\title{
Polya and GeoGebra : A dynamic approach to problem solving.
}

\author{
Alfonso Meléndez Acuña \\ Departamento de Matemáticas, Escuela Colombiana de Ingeniería, Bogotá, Colombia. \\ For correspondence: alfonso.melendez@escuelaing.edu.co
}

\begin{abstract}
Problem solving has been investigated in mathematics education for more than 60 years ago with the pioneering work of George Polya ( Polya , 1965). The four steps he proposes : understand the problem , devise a plan, carry it out and look back still apply in many instances. In recent years the emergence of dynamic mathematicss has enhanced the student creative and heuristics skills allowing the immediate construction of mathematical objects, their relationships and their interactive manipulation (Christou, Mousoulides, Pittalis \& Pitta - Pantazi , 2005), this has generated great interest in building dynamic learning scenarios to support the different stages of problem solving. In this paper a learning approach to problem solving activity using GeoGebra, one of the most popular tools of dynamic mathematics is presented.
\end{abstract}

Keywords: dynamic mathematics, GeoGebra, problem solving, Geometry

\section{Introduction}

"First, guess; then prove... Finished mathematics consists of proofs, but mathematics in the making consists of guesses" (Pólya, 1966)

The GeoGebra environment is very useful as a teaching and conceptual tool at different stages of the problem solving methodology suggested by George Polya . Given a mathematical problem it is possible to understand it through the construction of a dynamic model, where their objects and relationships are explicit, using this model the student can formulate conjectures and a plan to validate them. To carry out the plan the so called visual tests that verify the correctness of the solution to the problem can be constructed. Finally, looking back you can discover new properties of the objects involved in the problem statement, consider the following geometric problem:

Construct a circle with center $C$, define two points on it located in the bottom of the circle ( $A$ and $B$ ). Join $A$ to $C$ and $B$ to $C$, forming a central angle $(\angle A C B)$.

Define a point $D$ on the top of the circle, join $A$ with $D$ and $B$ to $D$, forming an inscribed angle $(<A C B)($ Figure 1$)$.

- What is the relationship between the two angles?

- Is this relationship preserved if $A, B$ and $D$ change of position on the circle?

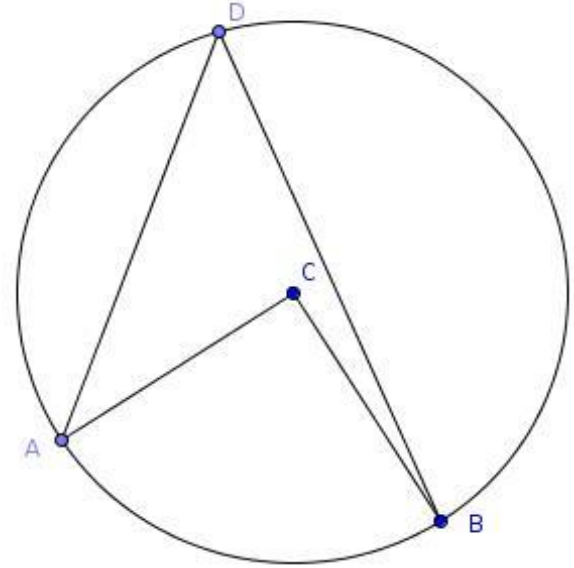

Figure 1 
Step 1. Understanding the problem

For helping understand the problem, a dynamic model is useful (Figure 2) to:

1) Dynamically visualize the problem statement.

2) Feed the model with measures of segments and angles that help formulate conjectures.

3) Analyze multiple instances of the problem "dragging" the different elements (point, circle).

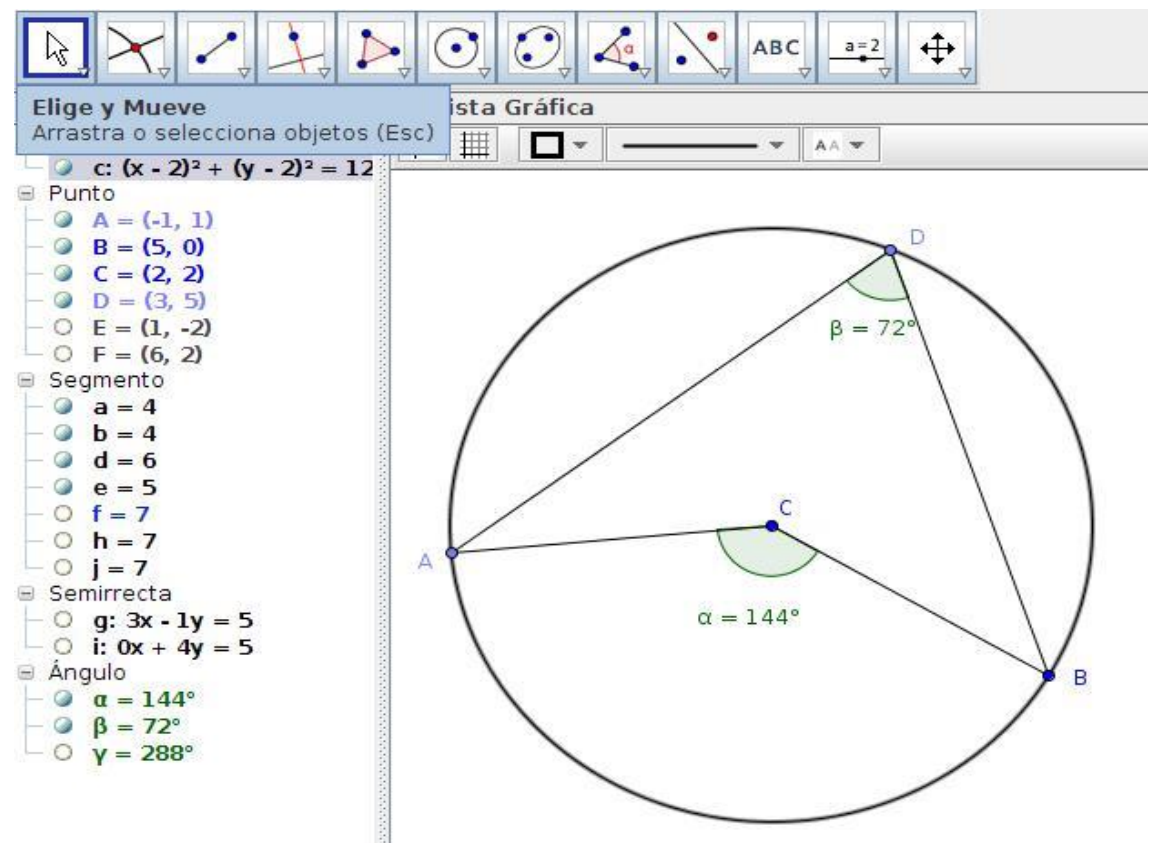

Figure 2

Step 2. Design a plan

Once the dynamic model is constructed (if necessary, with teacher's help) the student can easily conjecture about the relationship between the angles. In this case the central angle seems twice the exterior angle if the point $\mathrm{D}$ is on the top of the circle, but this relationship does not hold if $\mathrm{D}$ point $\mathrm{D}$ is placed below points $\mathrm{A}$ and $\mathrm{B}$ (Figure 3).

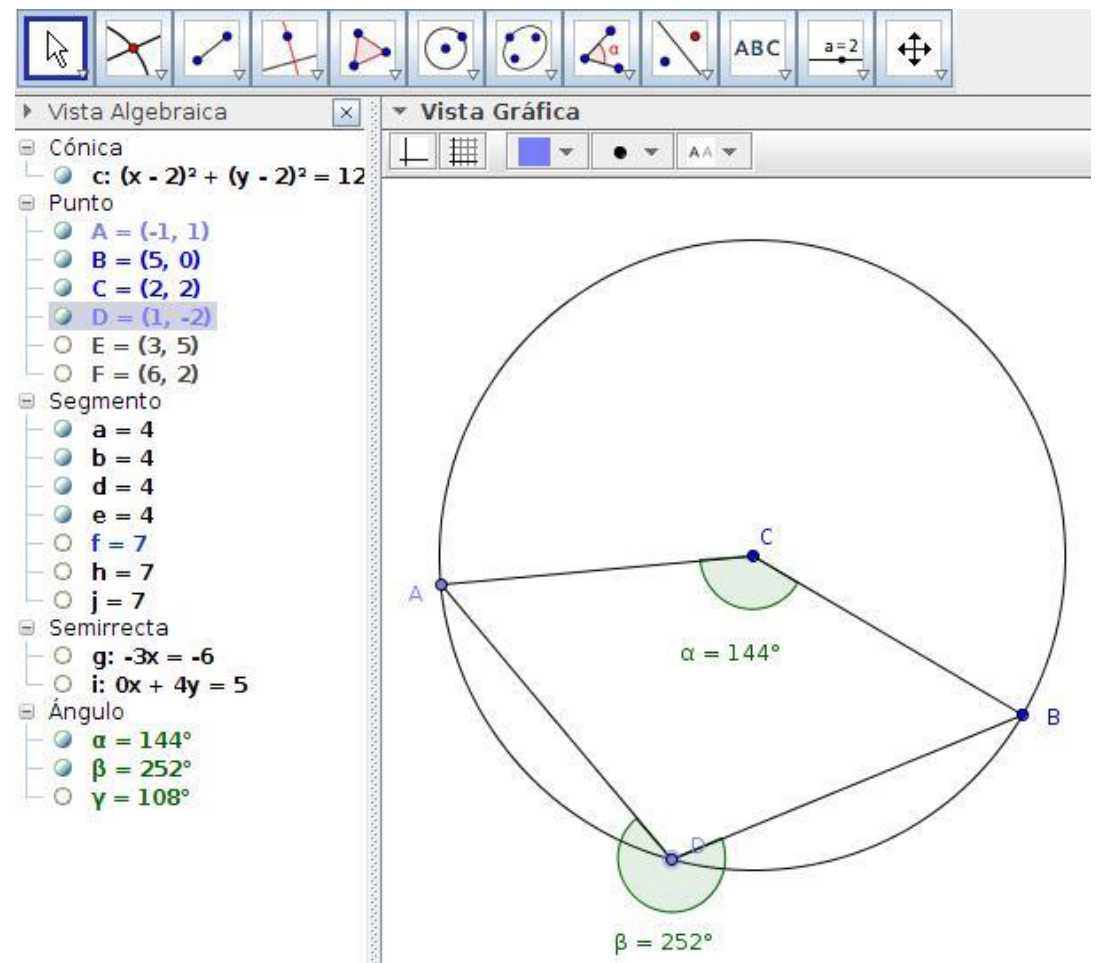

Figure 3 
Step 3. Carry out the plan

The dynamic model allows analyzing special cases that lead to understanding the overall relationship between the angles, an idea that may arise is placing A and D diametrically opposite (Figure 4).

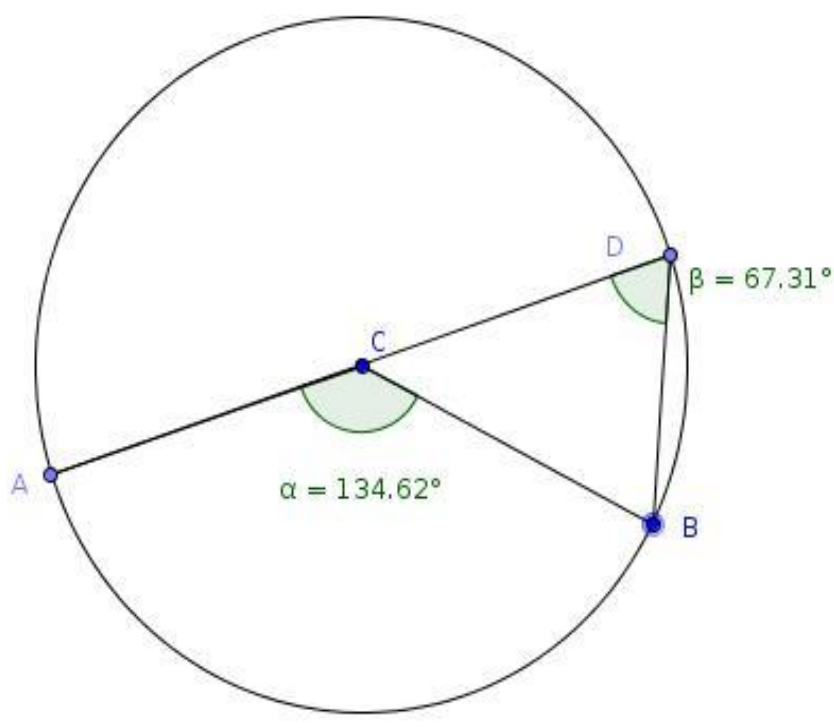

Figure 4

It is easy to see that triangle $B C D$ is an isosceles triangle $(B C=B D)$ and the central angle at $C$ is an exterior angle, so $\alpha=2 \beta$. How to go from here to the general case? By considering the diameter AD and draging the point $D$ we obtain a figure where the student can easily get the proof $\alpha=2 \beta$ by considering the isosceles triangles $\mathrm{ACD}$ and $\mathrm{BCD}$ and noting that angles $\angle \mathrm{ACE}$ and $\angle \mathrm{ECB}$ are respectively exterior angles, thus solving the problem in case point $\mathrm{D}$ is at the top (figure 5).

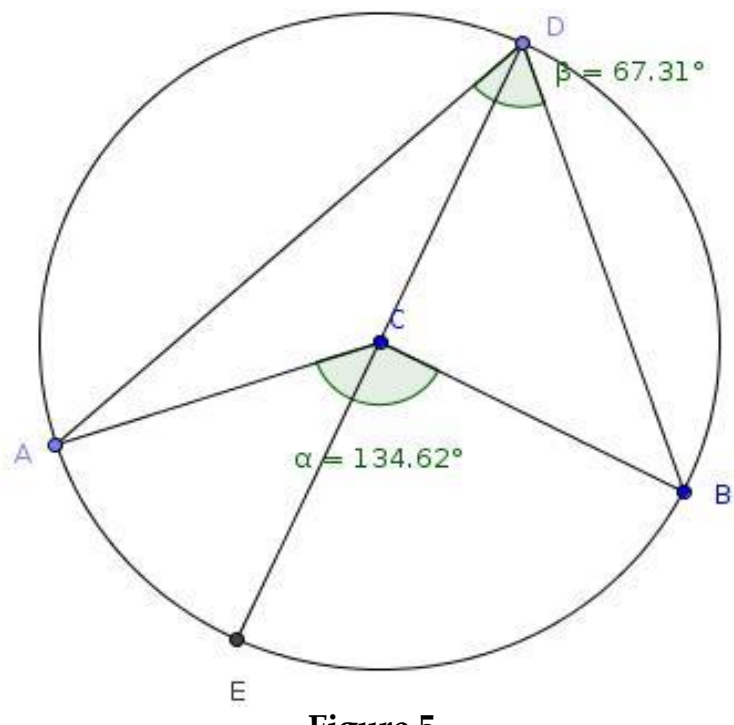

Figure 5

Step 4. Looking back

Looking back at Step 3 we can find the solution in the case where the point $\mathrm{D}$ is located at the bottom. A special case that can be useful is when A and B are diametrically opposite. In this case the angle at $\mathrm{D}$ is a right angle (this result is known as Thales' theorem). By building with GeoGebra the symmetric point to $\mathrm{D}$ with respect to the center $\mathrm{C}\left(\mathrm{D}^{\prime}=\right)$ and joining $\mathrm{A}$ and $\mathrm{B}$ with $\mathrm{D}^{\prime}$ we obtain the following figure (Figure 6). 


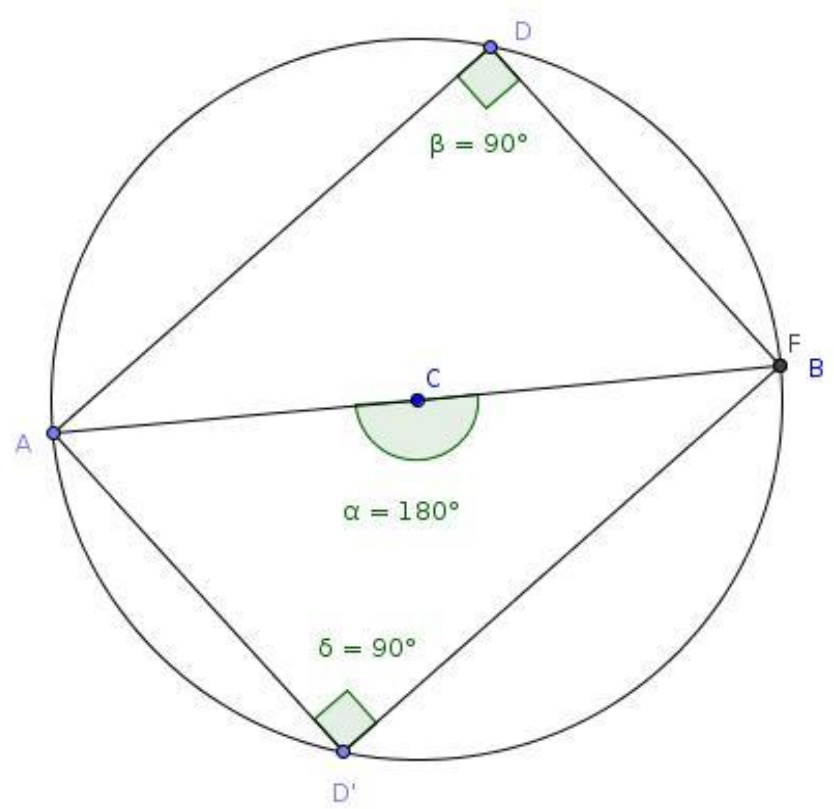

Figure 6

Here we see that angles at $\mathrm{D}$ and $\mathrm{D}$ 'are right angles and by dragging $\mathrm{A}$ and $\mathrm{B}$ the sum of these two angles ( $\beta$ and $\delta$ ) is always equal to two right angles (Figure 7).

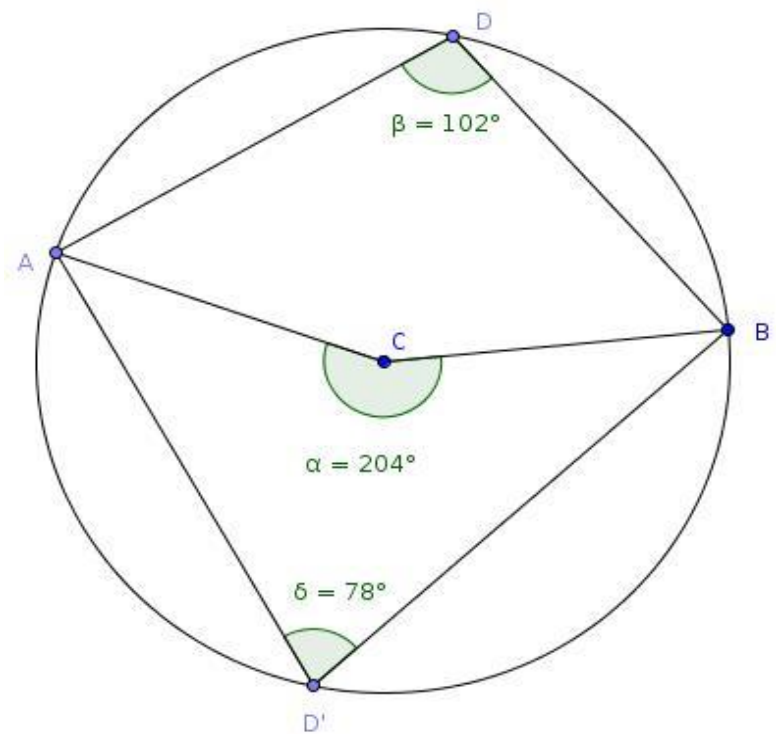

Figure 7

The relationship of the angles in this case is $\alpha=180^{\circ}-\beta / 2$.

\section{Conclusions}

- Dynamic Mathematics allow visualize mathematical objects in motion and develop a visual understanding of mathematical concepts and the relationships between them (Moreno-Eye Bolt \& Kaput,2008)

- Once an understanding of the objects and their relationship develops, follows the stage of "collect" data to test conjectures by dragging different model objects in GeoGebra.

- GeoGebra is a suitable working environment for solving mathematical problems. 


\section{References}

Christou, C., Mousoulides, N., Pittalis M., \& Pitta-Pantazi, D. (2005). Problem solving and problem posing in a dynamic geometry environment. The Montana Mathematics Enthusiast, 2(2), 125-143.

Moreno-Armella, L., Hegedus, Stephen J. \& Kaput, J. J. (2008). From Static to Dynamic Mathematics: Historical and Representational Perspectives. Educational Studies in Mathematics, 68(2), 99-111.

Pólya, G. (1965). Cómo plantear y resolver problemas. México: Trillas.

Radford, L. (1994) La enseñanza de la demostración: aspectos teóricos y prácticos, Educación Matemática, 6, 3, 21-51.s, 